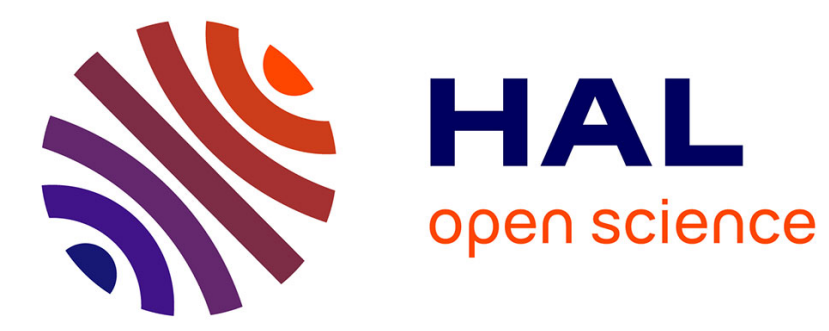

\title{
Prise en charge interventionnelle de la fuite mitrale
}

\author{
C. Delhomme, N. Tence, N. Karam
}

\section{To cite this version:}

C. Delhomme, N. Tence, N. Karam. Prise en charge interventionnelle de la fuite mitrale. Annales de Cardiologie et d'Angéiologie, 2019, 68, pp.468 - 473. 10.1016/j.ancard.2019.09.023 . hal-03488373

\section{HAL Id: hal-03488373 \\ https://hal.science/hal-03488373}

Submitted on 21 Dec 2021

HAL is a multi-disciplinary open access archive for the deposit and dissemination of scientific research documents, whether they are published or not. The documents may come from teaching and research institutions in France or abroad, or from public or private research centers.
L'archive ouverte pluridisciplinaire HAL, est destinée au dépôt et à la diffusion de documents scientifiques de niveau recherche, publiés ou non, émanant des établissements d'enseignement et de recherche français ou étrangers, des laboratoires publics ou privés.

\section{다)(1) $(5$}

Distributed under a Creative Commons Attribution - NonCommerciall 4.0 International 


\section{Prise en Charge Interventionnelle de la Fuite Mitrale \\ Percutaneous Treatment of Mitral Regurgitation}

Clemence Delhomme, MD ${ }^{1,2}$; Noemie Tence, MD, MPH ${ }^{1,2}$; Nicole Karam, MD, PhD ${ }^{1,2,3,4}$

${ }^{1}$ Département de Cardiologie, Hôpital Européen Georges Pompidou, AP-HP, Paris, France

${ }^{2}$ Université Paris Descartes, Paris, France

${ }^{3}$ INSERM Unité 970, Equipe 4, Paris, France

\section{Auteur correspondant:}

Nicole KARAM, MD, PHD

Hôpital européen Georges-Pompidou

INSERM Unité 970, Equipe 4

20, rue Leblanc, 75015 Paris, France

Tel: 0156093614 - Fax: 0156092664

Mail: nicole_karam@hotmail.com 


\title{
Résumé
}

L'insuffisance mitrale (IM) est actuellement la valvulopathie la plus fréquente et la deuxième valvulopathie la plus opérée en Europe. Environ $50 \%$ des patients présentant une IM sévère ne sont pas opérés, en dépit du mauvais pronostic associé à l'absence d'intervention, en raison de comorbidités et/ou d'âge avancé. Depuis quelques années, le traitement percutané, à type de remplacement ou surtout de réparation, des valves mitrales s'est développé, permettant de prendre en charge ces patients récusés de chirurgie, mais aussi les patients à haut risque chirurgical. La réparation mitrale percutanée bord-à-bord est actuellement la technique la plus utilisée et la seule reconnue dans les recommandations européennes pour la prise en charges des valvulopathies. Nous revoyons dans cet article, les différentes stratégies de traitement percutané de l'IM, ainsi que la place et le niveau de preuve pour leur utilisation.

Mots clés: Fuite Mitrale; traitement percutané; réparation mitrale; remplacement mitral

\begin{abstract}
Mitral regurgitation (MR) is currently the most frequent valvular heart disease, and the second most operated valve in Europe. Around 50\% of patients presenting severe MR are denied surgery, despite the adverse prognosis associated with the absence of treatment, due to comorbidities and/or advanced age. During the previous years, percutaneous treatment of MR, whether by replacement or, more frequently, by repair, has been developed, providing an alternative for those patients who are deemed at prohibitive, but also, high surgical risk. Percutaneous edge-to-edge repair is currently the most frequently used technique, and is the only one recommended by the European Guidelines for Valvular Heart Disease Management. In the current article, we review the different strategies for MR repair, as well as the indications and level of evidence for their use.
\end{abstract}

Keywords: Mitral regurgitation; percutaneous treatment; mitral repair; mitral replacement 


\section{1- Cas Clinique}

Une femme de 89 ans est adressée en consultation pour un deuxième avis concernant une dyspnée stade III de la New York Heart association (NYHA). Elle est hypertendue, en fibrillation auriculaire (FA) permanente, et suivie depuis 2 ans pour une insuffisance mitrale (IM) sévère organique sur prolapsus du feuillet postérieur, estimée inopérable devant l'âge avancé de la patiente et ses comorbidités.

Les échographies cardiaques trans-thoracique et trans-oesophagienne retrouvent un ventricule gauche $(\mathrm{VG})$ dilaté avec fraction d'éjection du ventricule gauche (FEVG) à 67\%, une IM sévère par prolapsus sur rupture de cordage en P2 avec une surface d'orifice régurgitant $(\mathrm{SOR})$ à $0.8 \mathrm{~cm} 2$ et une hypertension artérielle pulmonaire sévère avec des pressions artérielles pulmonaires systoliques (PAPs) estimées à 70mmHg (Figure 1A et 1B). L'EuroSCORE 2 est calculé à $7.5 \%$ et le risque opératoire considéré important chez cette patiente âgée, en surpoids et porteuse d'une HTAP.

Une procédure de réparation mitrale percutanée avec un MitraClip ${ }^{\circledR}$ est donc réalisée avec mise en place d'un clip en $\mathrm{A} 2 \mathrm{P} 2$, aboutissant à une fuite mitrale résiduelle minime en fin de procédure, avec un gradient transmitral en fin de procédure à $2 \mathrm{mmHg}$. La patiente est rentrée à domicile 3 jours après la procédure. Au contrôle à 6 mois, elle est asymptomatique en stade I de la NYHA, toujours en FA. L'échographie retrouve une normalisation des dimensions du VG avec une FEVG 65\%. L'IM résiduelle est estimée minime (Figure 1C et 1D). Les PAPs sont normalisées.

\section{2- Introduction}

Avec une fréquence croissante, l'IM constitue actuellement la valvulopathie la plus fréquente en Europe, et la seconde valvulopathie la plus opérée après le rétrécissement aortique.[1][2] Elle est associée à un pronostic défavorable lors qu'elle est sévère avec une surmortalité en l'absence de traitement.[3] Les différents types d'IM sont décrits dans la classification de 
Carpentier (Figure 2).[4] En pratique, on distingue deux modalités d'IM, qui différent de par leurs mécanismes, leurs pronostics et leurs prises en charge. L'IM primaire est caractérisée par une anomalie de l'appareil mitral qui est responsable de l'IM, notamment dans le cadre de prolapsus par rupture de cordage, de perforation de feuillet mitral ou de restriction de feuillet dans le cadre d'une valvulopathie rhumatismale. Dans l'IM secondaire, la valve est anatomiquement normale, et l'IM est due à des anomalies du VG ou de l'oreillette gauche, soit par une dilatation de l'anneau mitral, soit par une restriction des feuillets à cause d'une traction par l'appareil sous valvulaire. Dans l'IM primaire sévère, le traitement de référence est la chirurgie, alors que dans l'IM secondaire, le bénéfice de la chirurgie est incertain. En pratique, $50 \%$ des patients présentant une IM sévère sont récusés de la chirurgie devant un risque opératoire estimé trop élevé.[5][6] Chez les patients présentant une IM sévère symptomatique avec signes d'insuffisance cardiaque, l'absence de thérapie de la valve mitrale est associée à des taux de mortalité à 1 et 5 ans de $20 \%$ et $50 \%$ respectivement. Parmi les survivants, le taux de patients admis pour insuffisance cardiaque est de $41 \%$ à 1 an et $90 \%$ à 5ans.[7] Chez les patients asymptomatiques, en l'absence de thérapie valvulaire, le taux de décès de toutes causes et de décès d'origine cardiovasculaire à 5 ans est de $22 \%$ et $14 \%$ respectivement.[8] Depuis quelques années, différentes techniques de réparation ou de remplacement valvulaire mitral par voie percutanée se sont développées, permettant ainsi de proposer une alternative thérapeutique, dans un premier temps, aux patients récusés de chirurgie, puis progressivement, aux patients à haut risque chirurgical.

\section{3- Stratégies de traitement percutané de l'IM}

Plusieurs stratégies de réparation ou remplacement mitral ont été proposées. Contrairement à la valve aortique où le remplacement percutané a permis de révolutionner la prise en charge des rétrécissement aortiques, le remplacement percutané de la valve mitral s'est heurté à plusieurs défis, notamment dus à la forme particulière de l'anneau en «D», l'absence de 
structure annulaire rigide, la moindre présence de calcifications et le risque d'obstruction de la chambre de chasse du VG. D'autre part, les études faites dans le cadre de la chirurgie cardiaque n'avaient pas montré de supériorité du remplacement par rapport à la réparation de la valve mitrale.[9] Actuellement, plusieurs prothèses percutanées sont en évaluation préclinique ou clinique, mais le nombre de remplacements mitraux réalisés reste faible par rapport à la réparation percutanée, excepté dans le cas particulier des dégénérescences de bioprothèses ou d'IM sur anneau chirurgical, où les prothèses de remplacement percutané aortique sont utilisées depuis plusieurs années avec de bons résultats.[10]

En termes de réparation, plusieurs techniques ont été utilisées, avec en tête de ligne, la réparation des feuilles (essentiellement avec le MitraClip ${ }^{\circledR}$, et plus récemment le Pascal®). Les annuloplasties directe (essentiellement avec le Cardioband ${ }^{\circledR}$ et le Mitralign ${ }^{\circledR}$ ) ou indirecte (Carillon®) sont également proposées, avec une expérience croissante, quoique plus limitée. La réparation des cordages est aussi proposée, principalement avec le Neochord®, qui nécessite un abord transapical, contrairement aux techniques précitées qui nécessitent uniquement un abord percutané, fémoral ou jugulaire.

La réparation mitrale bord-à-bord percutanée par MitraClip® est la seule technique validée dans les recommandations européennes sur les valvulopathies. Elle est actuellement la procédure de réparation mitrale percutanée la plus fréquemment réalisée dans le monde, avec plus de 100000 patients traités à ce jour. La procédure se déroule sous anesthésie générale, avec un guidage par échographie cardiaque trans-oesophagienne. Le principe, dérivé de la technique chirurgicale d'Alfieri, consiste à réunir ensemble par un clip le feuillet antérieur et le feuillet postérieur, au niveau de la zone principale d'IM. Plusieurs clips peuvent être nécessaires afin d'obtenir une réduction satisfaisante de la l'IM. La sélection des patients est un élément clé pour le succès de la procédure, et dépend de critères anatomiques permettant de vérifier la faisabilité (Figure 3) et cliniques, afin d'éviter la futilité. 


\section{4- IM primaire}

Dans le cas de l'IM primaire, les traitements médicamenteux ont uniquement pour but de contrôler les symptômes, mais ne peuvent pas diminuer la sévérité de l'IM. Une réparation ou un remplacement de la valve mitrale est le seul moyen d'éviter la dilatation progressive du VG et la dysfonction ventriculaire ultérieure. Les recommandations actuelles de la Société Européenne de Cardiologie retiennent une indication chirurgicale en cas d'IM IM primaire sévère chez les patients présentant asymptomatiques avec FEVG $\leq 60 \%$, un diamètre télésystolique du VG $\geq 45 \mathrm{~mm}$, une FA d'apparition récente ou une PAPs $>50 \mathrm{mmHg}$. $\mathrm{La}$ chirurgie peut aussi être envisagée chez les patients asymptomatiques si la probabilité de réparation mitrale est élevée, le risque chirurgical est bas, et les patients présentent un diamètre télésystolique $\mathrm{du} V \mathrm{VG} \geq 40 \mathrm{~mm}$ associé à flail ou à un volume de l'oreillette gauche $\geq$ $60 \mathrm{ml} / \mathrm{m}^{2}$. Chez les patients symptomatiques, la chirurgie est envisagée d'emblée si la FEVG est $>30 \%$. En cas de FEVG $\leq 30 \%$, un traitement médical doit être entrepris en première intention. En cas d'échec, la chirurgie sera envisagée en cas de probabilité élevée de réparation mitrale et de comorbidités faibles; sinon, le traitement médical doit être continué et le traitement percutané bord-à-bord peut être envisagé (Figure 4).[11]

La réparation mitrale percutanée par MitraClip® a d'abord été étudié dans l'IM primaire, avec l'étude randomisée Everest II qui a comparé la chirurgie mitrale à la réparation percutanée par MitraClip® chez 279 patients porteurs d'IM grade III ou IV. Les patients inclus dans cette étude étaient relativement jeunes $(67.3 \pm 12.8$ ans dans le groupe MitraClip®) et opérables, avec un score STS moyen à $5 \pm 4 \%$. La fraction d'éjection moyenne était de $60 \%$. Moins d'événements indésirables majeurs ont été observés dans le groupe interventionnel que dans le groupe chirurgical (15\% vs. $48 \%$ respectivement, $\mathrm{p}<0.001)$.[12] A 5ans, moins de patients étaient indemnes de l'end-point composite (décès, chirurgie mitrale, réopération ou $\mathrm{IM} \geq 3+$ ) dans le groupe interventionnel que dans le groupe chirurgical (44.2 
vs. $64.3 \%, \mathrm{P}=0.01)$. Cependant, au-delà de la première année, parmi les patients n'ayant pas présenté d'événement dans la première année, il n'y avait pas de différence dans le taux de l'end point primaire entre les patients traités par voie percutanée $(69 \%)$ et ceux traités par chirurgie $(75 \%, \mathrm{P}=0.55)$. En effet, la différence de composite à 5ans était essentiellement due au taux d'IM $\geq 3+$ et à la nécessité de réintervention, sachant que $78 \%$ des chirurgies dans le groupe interventionnel ont eu lieu dans les 6 premiers mois après le clip. La mortalité à 5 ans était similaire dans les 2 groupes (20.8\% dans le bras interventionnel vs. $26.8 \%$ dans le bras chirurgical, $\mathrm{P}=0.4)$.[13]

\section{5- IM secondaire}

Dans l'IM secondaire, les structures de la valve mitrale sont intactes et l'IM résulte d'une dilatation du ventricule ou de l'oreillette gauche, à l'origine d'un défaut de coaptation des feuillets mitraux. L'apparition d'une IM secondaire sévère est aussi associée à une augmentation de la mortalité [14]. Le traitement médicamenteux est le traitement de première intention, basé sur les diurétiques et le traitement de l'insuffisance cardiaque. Une resynchronisation peut être indiquée en complément si les patients restent symptomatiques, en présence d'un bloc de branche gauche. Dans le cas de l'IM secondaire, le bénéfice de la chirurgie est mitrale est incertain, car elle n'a pas démontré de bénéfice sur la mortalité, en raison du risque chirurgical lié à la dysfonction du VG, ainsi qu'à la présence d'une pathologie cardiaque extra-valvulaire qui évolue pour son propre compte. Les recommandations actuelles de la Société Européenne de Cardiologie préconisent une chirurgie de la valve mitrale en cas d'IM sévère avec indication à une revascularisation chirurgicale, associée à une FEVG > 30\% (recommandation de classe I, niveau d'évidence C), ou $<30 \%$ avec viabilité documentée chez un patient symptomatique (recommandation de classe IIa, niveau d'évidence C). En l'absence d'indication à une revascularisation, la chirurgie est réservée aux patients ayant une FEVG >30\% ayant une symptomatologie réfractaire au 
traitement médical optimal et avec un faible risque chirurgical (recommandation de classe IIb, niveau d'évidence C). Elle peut aussi être discutée en cas de FEVG < 30\% après avoir envisagé les possibilités de greffe et d'assistance ventriculaire (recommandation de classe IIb, niveau d'évidence C), mais dans ce cas, la réparation percutanée bord-à-bord peut aussi être discutée. Le traitement percutanée par réparation bord-à-bord peut aussi être envisagé dans l'IM secondaire si la FEVG est > 30\%, sans indication à une revascularisation, chez un patient symptomatique et ayant un risque chirurgical jugé non bas (recommandation de classe IIb, niveau d'évidence C; Figure 4).[11]

Le bénéfice de la réparation mitrale percutanée par MitraClip a été évalué dans deux études randomisées parues en 2018. MITRA-FR, une étude française, contrôlée et randomisée, a comparé le traitement médical optimal isolé à la réparation mitrale percutanée par Mitraclip® associée au traitement médical optimal, chez les patients symptomatiques, ayant une IM secondaire sévère avec une FEVG entre 15 et $40 \%$ et une surface de l'orifice régurgitant $>20 \mathrm{~mm}^{2}$ ou un volume régurgitant $>30 \mathrm{~mL}$. Malgré un taux de succès d'implantation du Mitraclip® de 95\%, l'étude n’a pas montré de différence significative entre les deux groupes en terme de décès toute cause ou de réhospitalisation pour insuffisance cardiaque à un an (51,3\% dans le groupe traitement médical vs. 54,6\% dans le groupe intervention, $\mathrm{p}=0,53)$ ou à 2 ans $(67.1 \%$ vs. 63.8 respectivement, Hazard ratio, 1.01; intervalle de confiance à 95\%, 0.77 to 1.34.[15][16] COAPT, une étude américaine, a également évalué le bénéfice du MitraClip en plus du traitement optimal chez les patients ayant une FEVG entre 20 et 50\%, avec IM plus sévère que dans MITRA-FR (SOR > 30mm² ou volume régurgité > $45 \mathrm{ml}$ ). L'essai a montré une différence significative en faveur du MitraClip® sur les réhospitalisations pour insuffisance cardiaque dans les 24 mois suivant la randomisation (35,8\% dans le groupe MitraClip ${ }^{\circledR}$ versus $67,9 \%$ dans le groupe traitement médical, $\mathrm{p}<0,001)$.[17] 
Ces différences de résultats entre les deux études peuvent être expliquées par une différence dans les critères d'inclusion et d'exclusion de la population mais aussi dans la définition échographique de sévérité de l'IM secondaire entre les recommandations européennes et américaines. L'IM était moins sévère dans Mitra-FR avec un SOR moyen à $31 \mathrm{~mm}^{2}$ vs. $41 \mathrm{~mm}^{2}$ dans COAPT, alors que le volume télédiastolique du VG était plus élevé (136.2vs. $\left.101 \mathrm{ml} / \mathrm{m}^{2}\right)$, témoignant d'une atteinte ventriculaire plus sévère et au premier plan par rapport à l'IM.[18] Ces différences entre Mitra-FR et COAPT, en termes de caractéristiques de base et de bénéfice de la réparation mitrale percutanée, nous permettent donc de distinguer une population cible, qui bénéficierait du traitement percutané, et une population chez qui l'atteinte myocardique est au premier plan, devant l'IM, rendant l'intervention percutanée moins intéresssante.

L'intérêt de la réparation percutanée de l'IM secondaire a été souligné récemment avec le système de réparation percutanée Carillon ${ }^{\circledR}$ dans une étude randomisée publiée récemment, REDUCE FMR, qui a montré une diminution des volumes ventriculaires et du volume régurgitant après annuloplastie indirecte par Carillon ${ }^{\circledR}$ par rapport aux patients ayant eu uniquement un traitement médical optimal. Les patients traités par Carillon® ont également présenté une amélioration de leur test de marche à 6 minutes, une amélioration qui n'a pas été observée dans le groupe témoin.[19]

\section{Conclusion}

De nombreux progrès ont eu lieu durant les dernières années en termes de traitement percutané des IM. Les différentes techniques développées permettent aujourd'hui de prendre en charge des IM récusées de chirurgie ou à haut risque chirurgical. La bonne sélection par une équipe de valve de type "heart valve team" incluant des cardiologues interventionnels, mais aussi des chirurgiens cardiaques, des échographistes, des spécialistes d'insuffisance 
cardiaque, des anesthésistes et, si besoin, des gériatres, est capitale afin de sélectionner les patients qui seraient les plus à même à profiter de ces techniques. 


\section{Conflits d'intérêts:}

NK : Abbott Vascular (honoraire de consultante) et Edwards Scientific (bourse de recherche);

CD et NT: aucun conflit d'intérêt 


\section{References}

[1] Nkomo VT, Gardin JM, Skelton TN, Gottdiener JS, Scott CG, Enriquez-Sarano M. Burden of valvular heart diseases: a population-based study. Lancet 2006;368:1005-11. doi:10.1016/S0140-6736(06)69208-8.

[2] Iung B. A prospective survey of patients with valvular heart disease in Europe: The Euro Heart Survey on Valvular Heart Disease. European Heart Journal 2003;24:1231-43. doi:10.1016/S0195-668X(03)00201-X.

[3] Trichon BH, Felker GM, Shaw LK, Cabell CH, O'Connor CM. Relation of frequency and severity of mitral regurgitation to survival among patients with left ventricular systolic dysfunction and heart failure. The American Journal of Cardiology 2003;91:538-43. doi:10.1016/S0002-9149(02)03301-5.

[4] Carpentier A. Cardiac valve surgery--the "French correction." J Thorac Cardiovasc Surg 1983;86:323-37.

[5] Mirabel M, Iung B, Baron G, Messika-Zeitoun D, Detaint D, Vanoverschelde J-L, et al. What are the characteristics of patients with severe, symptomatic, mitral regurgitation who are denied surgery? European Heart Journal 2007;28:1358-65. doi:10.1093/eurheartj/ehm001. [6] Joint Task Force on the Management of Valvular Heart Disease of the European Society of Cardiology (ESC), European Association for Cardio-Thoracic Surgery (EACTS), Vahanian A, Alfieri O, Andreotti F, Antunes MJ, et al. Guidelines on the management of valvular heart disease (version 2012). Eur Heart J 2012;33:2451-96. doi:10.1093/eurheartj/ehs109.

[7] Goel SS, Bajaj N, Aggarwal B, Gupta S, Poddar KL, Ige M, et al. Prevalence and outcomes of unoperated patients with severe symptomatic mitral regurgitation and heart failure: comprehensive analysis to determine the potential role of MitraClip for this unmet need. J Am Coll Cardiol 2014;63:185-6. doi:10.1016/j.jacc.2013.08.723. 
[8] Enriquez-Sarano M, Akins CW, Vahanian A. Mitral regurgitation. Lancet 2009;373:1382-94. doi:10.1016/S0140-6736(09)60692-9.

[9] Acker MA, Parides MK, Perrault LP, Moskowitz AJ, Gelijns AC, Voisine P, et al. Mitral-valve repair versus replacement for severe ischemic mitral regurgitation. N Engl J Med 2014;370:23-32. doi:10.1056/NEJMoa1312808.

[10] Guerrero M, Salinger M, Pursnani A, Pearson P, Lampert M, Levisay J, et al. Transseptal transcatheter mitral valve-in-valve: A step by step guide from preprocedural planning to postprocedural care. Catheter Cardiovasc Interv 2018;92:E185-96. doi:10.1002/ccd.27128.

[11] Baumgartner H, Falk V, Bax JJ, De Bonis M, Hamm C, Holm PJ, et al. 2017 ESC/EACTS Guidelines for the management of valvular heart disease. Eur Heart $\mathbf{J}$ 2017;38:2739-91. doi:10.1093/eurheartj/ehx391.

[12] Feldman T, Foster E, Glower DD, Kar S, Rinaldi MJ, Fail PS, et al. Percutaneous Repair or Surgery for Mitral Regurgitation. New England Journal of Medicine 2011;364:1395-406. doi:10.1056/NEJMoa1009355.

[13] Feldman T, Kar S, Elmariah S, Smart SC, Trento A, Siegel RJ, et al. Randomized Comparison of Percutaneous Repair and Surgery for Mitral Regurgitation: 5-Year Results of EVEREST II. J Am Coll Cardiol 2015;66:2844-54. doi:10.1016/j.jacc.2015.10.018.

[14] Goliasch G, Bartko PE, Pavo N, Neuhold S, Wurm R, Mascherbauer J, et al. Refining the prognostic impact of functional mitral regurgitation in chronic heart failure. European Heart Journal 2018;39:39-46. doi:10.1093/eurheartj/ehx402.

[15] Obadia J-F, Messika-Zeitoun D, Leurent G, Iung B, Bonnet G, Piriou N, et al. Percutaneous Repair or Medical Treatment for Secondary Mitral Regurgitation. New England Journal of Medicine 2018;379:2297-306. doi:10.1056/NEJMoa1805374.

[16] Iung B, Armoiry X, Vahanian A, Boutitie F, Mewton N, Trochu J-N, et al. 
Percutaneous Repair or Medical Treatment for Secondary Mitral Regurgitation: Outcomes at 2 years. Eur J Heart Fail 2019. doi:10.1002/ejhf.1616.

[17] Stone GW, Lindenfeld J, Abraham WT, Kar S, Lim DS, Mishell JM, et al. Transcatheter Mitral-Valve Repair in Patients with Heart Failure. New England Journal of Medicine 2018;379:2307-18. doi:10.1056/NEJMoa1806640.

[18] Praz F, Grasso C, Taramasso M, Baumbach A, Piazza N, Tamburino C, et al. Mitral regurgitation in heart failure: time for a rethink. European Heart Journal 2019;40:2189-93. doi:10.1093/eurheartj/ehz222.

[19] Witte KK, Lipiecki J, Siminiak T, Meredith IT, Malkin CJ, Goldberg SL, et al. The REDUCE FMR Trial: A Randomized Sham-Controlled Study of Percutaneous Mitral Annuloplasty in Functional Mitral Regurgitation. J Am Coll Cardiol HF 2019:1132. doi:10.1016/j.jchf.2019.06.011. 


\section{Légende des figures}

Figure 1 : Evaluation de la valve mitrale avant et 6 mois après la procédure. On note une fuite mitrale initiale sévère avant la procédure (A et $B$ ) avec un prolapsus de $\mathrm{P} 2$, réduite après traitement par MitraClip $($ avec une fuite résiduelle minime à modérée à 6 mois $(\mathrm{C}$ et D)

Figure 2: Classification de Carpentier pour les fuites mitrales. Le Type 1 est caractérisé par un mouvement normal des feuillets, le type 2 par un mouvement excessif des feuillets et le type 3 par une restriction du mouvement des feuillets en diastole (IIIa) ou en systole (IIIb).

Figure 3 : Critères de faisabilité anatomique de la réparation Mitrale percutanée par MitraCLip®. Les items présentés en gras correspondent à l'anatomie optimale pour une réparation bord-à-bord, alors que les items en italiques correspondent à une anatomie non optimale mais où la procédure peut être envisagée.

Figure 4 : Place du traitement percutané dans la prise en charge de la fuite mitrale. IM : insuffisance mitrale; FEVG: fraction d'éjection du ventricule gauche 

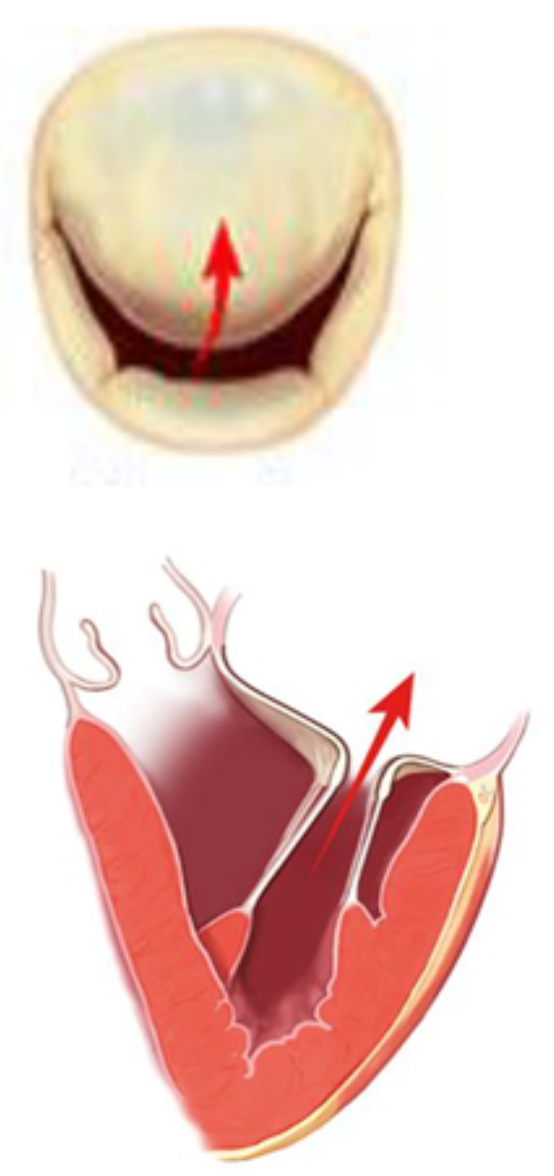

Type I
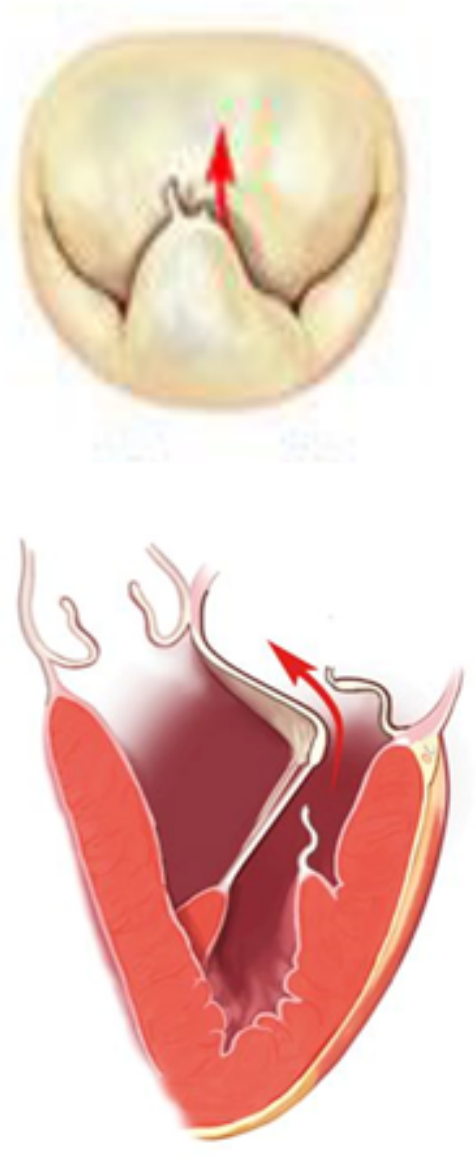

Type II
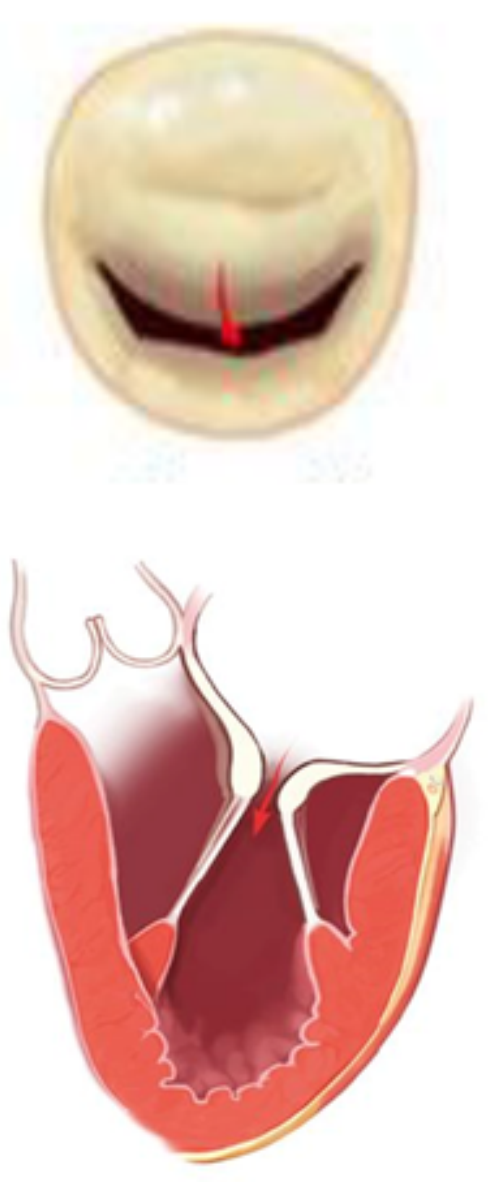

Type IIla
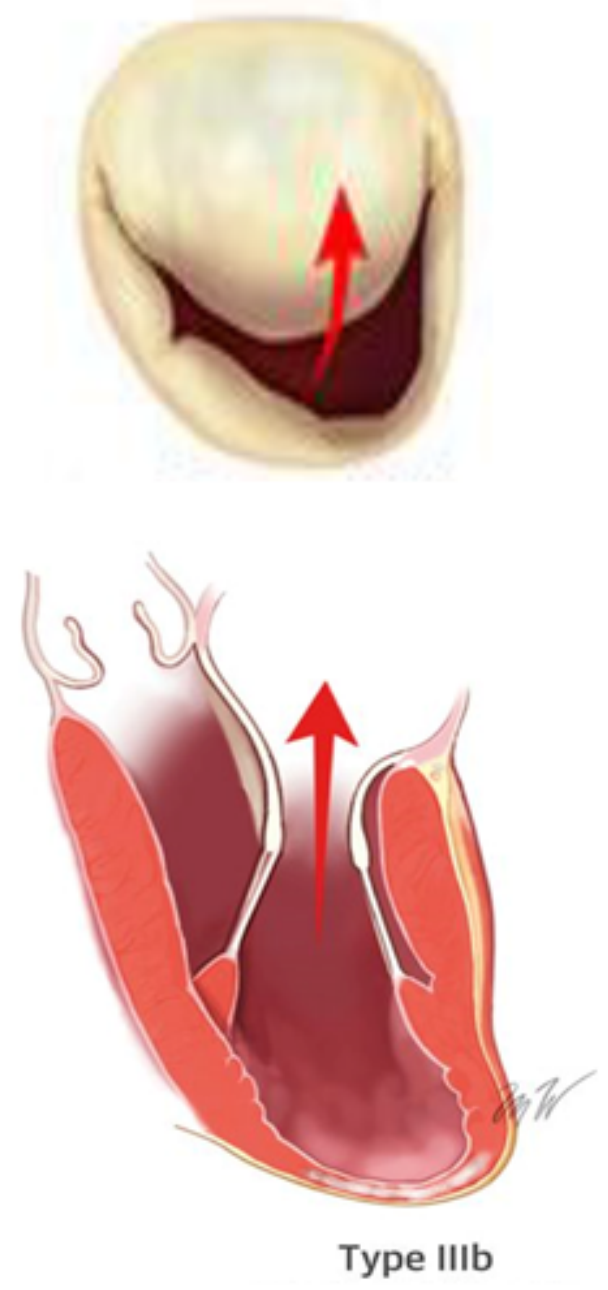


\section{Quelque soit le type d'IM}

- Fuite centrale - segment 2 ou segments 1 et 3

- Surface valvulaire $>4 \mathrm{~cm}^{2}$ ou $3 \mathrm{~cm}^{2}$ avec mobilité résiduelle acceptable des feuillets

- Longueur de la partie mobile de la petite valve $>10 \mathrm{~mm}$ ou entre 7 et $10 \mathrm{~mm}$

- Epaisseur de feuillets $<5 \mathrm{~mm}$

- Absence de calcification des feuillets ou calcification en dehors de la zone de grasp

\section{IM primaire :}

Profondeur de prolapsus

$<10 \mathrm{~mm}$

Largeur de prolapsus $<15 \mathrm{~mm}$

Ou $>15 \mathrm{~mm}$ avec possibilité de plusieurs clips

\section{IM fonctionnelle :}

Feuillets ayant une coaptation centrale $\geq 2 \mathrm{~mm}$

Hauteur de tenting $<11 \mathrm{~mm}$ $\mathrm{O} u>11 \mathrm{~mm}$ 
